\title{
Bruise Detection using NIR Hyperspectral Imaging for Strawberry (Fragaria $\times$ ananassa Duch.)
}

\author{
Masateru Nagata, Jasper G. TAlladA* and Taiichi Kobayashi* \\ Faculty of Agriculture, University of Miyazaki, \\ 1-1 Gakuen Kibanadai Nishi, Miyazaki 889-2192, Japan \\ * The United Graduate School of Agricultural Sciences, Kagoshima University, \\ 1-21-24 Korimoto, Kagoshima 890-0065, Japan
}

(Received December 19, 2005)

\begin{abstract}
Bruising in fruits and its detection is a great concern for food safety to the consumers, and for incurrence of economic losses to industry. This research was aimed at developing techniques for detection of compression bruises in 'Akihime' variety strawberries (Fragaria $\times$ ananassa Duch.) using near infrared (NIR) hyperspectral imaging. Using 120 fruit samples at $70-80 \%$ ripe and full-ripe levels of maturity that had received six levels of bruising force $(0 \mathrm{~N}, 0.5 \mathrm{~N}, 1.0 \mathrm{~N}$, $1.5 \mathrm{~N}, 2.0 \mathrm{~N}$ and $3.0 \mathrm{~N}$ ), spectral images were taken from 650 to $1,000 \mathrm{~nm}$ at $5 \mathrm{~nm}$ wavelength intervals and from 0 to 4 days after bruising. Using stepwise linear discriminant analysis, optimal wavelengths of $825 \mathrm{~nm}$ and $980 \mathrm{~nm}$ were identified. The three judgment methods (linear discriminant analysis, normalized difference and artificial neural network) had performed equally well, while the normalized difference method was found to be more useful. Additionally, the extent of bruising damage detected in strawberries by the judgment method had markedly decreased with time, which elucidated temporal changes in bruising pattern while in storage.
\end{abstract}

Keywords : artificial neural network, compression bruising, linear discriminant analysis, normalized difference, strawberry

\section{INTRODUCTION}

Mechanical bruising in fruits is a great concern to the consumers in particular and the industry in general. Because they are a latent form of damage, bruises are normally discovered too late while causing a reduction of quality grade, loss of consumer's confidence due to concern for food safety, and incurrence of economic losses. Strawberries (Fragaria $\times$ ananassa Duch.) are normally harvested ripe and so are very prone to bruising (Hancock, 1999). Compression bruising is easily incurred during strawberry manual harvesting and post-harvest operations. The detection of bruises is challenging because the damage occurs beneath the peel and is complicated by factors such as severity, variety of fruit, duration of occurrence, and pre- and post-harvest operations. Because of this, detection by humans would be very limited if not impossible particularly in an on-line grading operation. Common color machine vision techniques may be useful for detection of some major defects in fruits, but they are not very reliable for detecting latent damages such as bruising because of very small and inconsistent color difference between the bruised and non-bruised tissues.

Corresponding author: Masateru Nagata, fax : +81-985-58-7246,

e-mail : nagatam@cc.miyazaki-u.ac.jp 
Several researches had tackled the bruise detection problem in fruits. Spectroscopy was found useful for the detection of bruises in apples (Xing et al., 2003; Upchurch et al., 1994). As explained by Kim et al. (2001) and $\mathrm{Lu}$ (2003), a major limitation of this technique is that assessments are confined to small areas, thus it is not suitable for examination of whole fruits. Alternatively, spectral imaging can characterize both spatial and spectral variability of surface tissues; however it requires identification of optimal wavebands. In a review, Abbott (1999) had pointed out that at specific near infrared (NIR) wavelengths, bruises in apples and peaches can be detected; however, the chosen wavelength values differ between new and old bruises in apples because of consequent drying of injured tissues. Lu (2003) had used NIR hyperspectral imaging from 900 to 1,700 nm and principal component analysis and minimized noise fraction to detect new and old impact bruises in apples with an appreciable detection rate. Ariana et al. (2003) had used multi-spectral imaging in reflectance and fluorescence to detect six types of defects on three apples varieties. Using either linear discriminant or artificial neural network models, they achieved $100 \%$ accuracy for a two-class classification with artificial neural network showing better performance.

In peaches and apricots, Zwiggelaar et al. (1996) utilized spectral data from 500 to $1,600 \mathrm{~nm}$ and a simple statistical technique to identify effective wavelengths for bruise detection. From spectral images at $750 \mathrm{~nm}$ for apricots, and 930 and $970 \mathrm{~nm}$ for peaches, bruises were detected with a success rate of about $65 \%$. Using the same approach, Shrestha et al. (2002) used spectrometric readings from 600 to $1,000 \mathrm{~nm}$ from full-ripe strawberries receiving 0 to $4 \mathrm{~N}$ bruising force levels. Consequently, bruises were detected using 860 and $960 \mathrm{~nm}$ high-pass filters, and image subtraction. However, the results need confirmation using the hyperspectral imaging technique. Also, the effects of degree of ripeness maturity and storage time should be well considered.

This research aimed at developing a method for the detection of bruises on strawberries using hyperspectral imaging technology. The specific goals were to identify optimal wavebands for accurate detection of bruises at two ripeness maturity levels; to assess the effectiveness of several bruise judgment methods; and to evaluate the effects of storage time in the detection of new and old bruises.

\section{MATERIALS AND METHODS}

\section{Preparation of fruit samples}

From a local greenhouse cultivation farm, about 120 pieces of uniform L-sized strawberry fruits of 'Akihime' variety at two ripeness maturity levels ( $70-80 \%$ ripe and full-ripe based on coverage of red color on the fruit surface) were manually harvested early in the morning. They were then immediately brought to the laboratory for processing.

Bruises were carefully applied using a $25-\mathrm{mm}$ diameter ball tip driven in an Orientec Universal Testing Machine (UTM, STA-1150; Orientec Corporation, Japan) as shown in Fig. 1. Six levels of compression bruising forces $(0 \mathrm{~N}, 0.5 \mathrm{~N}, 1.0 \mathrm{~N}, 1.5 \mathrm{~N}, 2.0 \mathrm{~N}$, and $3.0 \mathrm{~N})$ were randomly assigned to a set of six samples of similar ripeness. The application of bruise force was performed in the following manner. A randomly selected fruit sample was placed on a rubber holder with the surface receiving the force approximately horizontal. It was then placed onto the UTM platform. After setting the level of bruising force, the ball tip was automatically driven at $20 \mathrm{~mm}$ $\min ^{-1}$ onto the fruit up until the force level was achieved, and it was then held motionless for about five s. The ball tip was then quickly lifted and the sample transferred to a random location on a six-cell sample holder.

\section{Hyperspectral imaging}

The hyperspectral imaging system shown in Fig. 2 was an illumination chamber covered with black curtains to exclude external light. It had a 14-bit Apogee AP2E (Apogee Instruments, Inc., Auburn, CA, USA) monochrome camera operated through the Maxim/DL software. A Varispec 


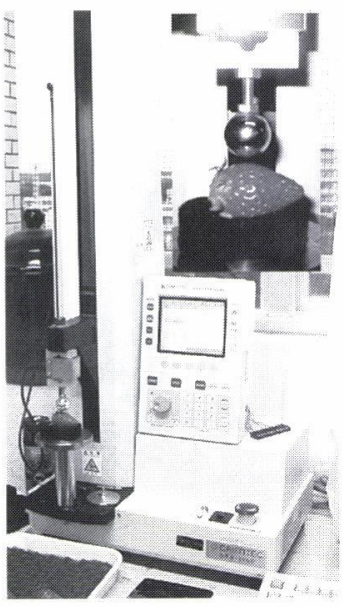

Fig. 1 The Orientec STA-1150 universal testing machine comprised the bruising set-up for strawberry using a 25-mm diameter ball tip (inset).

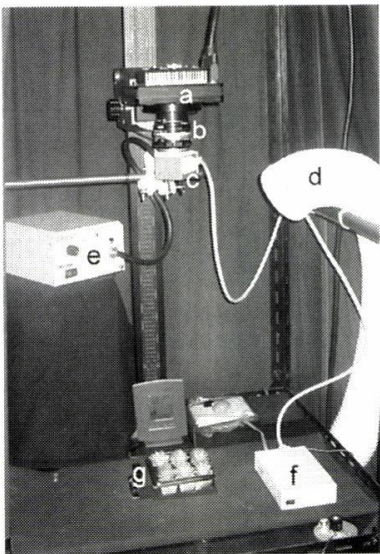

Fig. 2 The near-infrared hyperspectral imaging setup had the following parts: (a) Apogee AP2E camera, (b) Nikkor lens (c) CRI Varispec liquid cystal tunable filter, (d) cooler exhaust, (e) Dolan-Jenner FiberLite light source (f) Varispec controller box, and $(g)$ sample stage.

liquid crystal tunable filter (LCTF, Cambridge Research and Instrumentation, Inc., Woburn, MA, USA) Model VS-NIR-20-10 (650 to $1,100 \mathrm{~nm}, 10 \mathrm{~nm}$ bandwidth) was attached onto the Nikkor f/1.2 optical lens and controlled through the National Instruments Cris3 virtual instrument software. The Dolan-Jenner Fiber-Lite PL950 DC-regulated Illuminator (Dolan-Jenner Industries, St. Lawrence, MA, USA) had a 150-watt tungsten-halogen light bulb without the infrared filter. The fiber optic light guide was placed vertically above the stage. An air-conditioning cooler (TDBD12A, Toyotomi Corporation, Japan) maintained a relatively uniform temperature $\left(22^{\circ} \mathrm{C}\right)$ within the set-up.

\section{Spectral image acquisition}

Using custom-built Visual Basic software, full-sized spectral images of a set of fruits were automatically acquired from 650 to $1,000 \mathrm{~nm}$ at $5 \mathrm{~nm}$ wavelength intervals (71 images). The heights of the camera and light source were set at $72 \mathrm{~cm}$ and $54 \mathrm{~cm}$ respectively, and the light intensity adjusted to about 2,100 lux. Due to the wavelength aberration phenomenon, the focus of the camera was set at $650 \mathrm{~nm}$ and at $800 \mathrm{~nm}$ in each scan. The exposure time was varied from 0.70 to $7.25 \mathrm{~s}$ to account for the variable sensitivity of the system due to responsivity of the chargecoupled device (CCD) sensor, transmission efficiency of the LCTF and spectral distribution of the light as was earlier done by Evans et al. (1998), and Cogdill et al. (2004). Using the same acquisition settings, reference images from a $125 \times 125 \mathrm{~mm}$ Spectralon reference panel $(99 \%$ diffuse reflectance, SRT-99-050, Labsphere, Inc., North Sutton, NH, USA) and dark current images were also acquired with closed camera shutter at the start, after ten sets of samples and at the end of the imaging session.

\section{Spectral image data analysis}

Figure 3 shows the over-all pictorial flowchart of spectral data acquisition with the image analysis implemented using the Image Processing Toolbox of Matlab version 6.5 (The Mathworks, Inc., Natick, MA, USA). Since bruised tissues are easily distinguished from the $980 \mathrm{~nm}$ images by observation (see Fig. 5), 8-bit RGB images were derived to facilitate masking of pixels and masking of area-of-interest (AOI). Using the Microsoft Paint software, bruised tissue areas were 


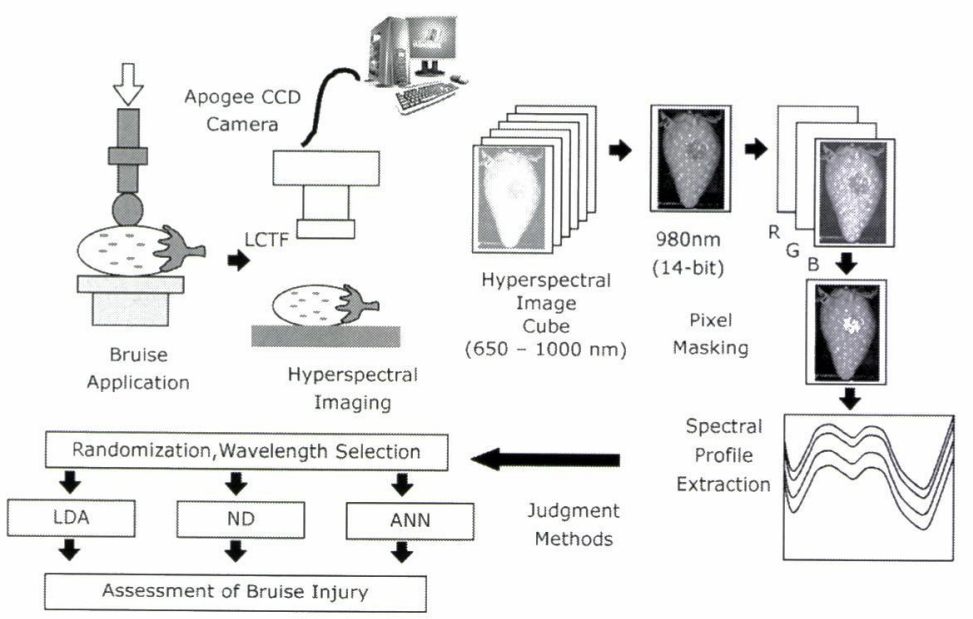

Fig. 3 Pictorial diagram of hyperspectral image data acquisition and analysis for identification of optimal wavelengths for bruise detection on strawberries.

manually marked with blue while normal tissues with red color. An AOI was also marked using a white 150-by-150 pixels square region that includes the bruised tissues whenever they were seen. A Matlab processing script applied flat field correction to sample images using the following formula:

$$
I_{\text {norm }}(x, y)=\frac{I_{\text {sample }}(x, y)-I_{\text {dark }}(x, y)}{I_{\text {referencee }}(x, y)-I_{\text {durk }}(x, y)} \cdot m
$$

where, $I_{\text {norm }}(x, y)$ is the normalized pixel value at pixel location $(x, y) ; I_{\text {sample }}(x, y), I_{\text {referennec }}(x, y), I_{\text {dart }}$ $(x, y)$ are the pixel values for the sample, reference and dark images, respectively, at the same location $(x, y)$; and $m$ is a factor based on the reference panel. Using the pixel mask image, normalized reflectance spectral data were extracted and some 400 points were randomly selected for statistical selection of wavelengths for judgment model development.

\section{Wavelength selection}

The stepwise linear discriminant analysis is a statistical technique used in machine learning to find a linear combination of features that would best classify objects into certain classes. Using SPSS version 11 (Statistical Product and Service Solutions, SPSS Inc., USA), a stepwise linear discriminant analysis was used to identify a limited set of wavelengths for discriminating bruised from non-bruised pixel points of randomly selected samples. Non-bruised pixels were selected from samples that had no bruising treatment (see Fig. 6) and from $0.5 \mathrm{~N}$ bruising force level of $70^{-}$ $80 \%$ ripe samples. On the other hand, bruised pixels were selected from $2.0 \mathrm{~N}$ and $3.0 \mathrm{~N}$ bruise force levels where bruises were quite discernible with certainty at $980 \mathrm{~nm}$ by observation. Additional samples were selected from the $1.5 \mathrm{~N}$ bruising force level of full-ripe maturity. Further from each selected sample, $60 \%$ of the 400 points were randomly selected for model development, and the remaining $40 \%$ for validation.

\section{Judgment model development}

In this study three judgment models were compared: (1) linear discriminant analysis, (2) normalized difference method and (3) back-propagation artificial neural network to detect bruised tissues within the AOI of each fruit. For linear discriminant analysis (LDA), the dicriminant function equation was used. From the same wavelengths, another judgment model was evaluated based on normalized difference (ND) images using the following equation (2): 


$$
I_{N D}(x, y)=\frac{I_{825}(x, y)-I_{980}(x, y)}{I_{825}(x, y)+I_{980}(x, y)}
$$

where, $I_{N D}(x, y)$ is the normalized difference image pixel value at pixel location $(x, y)$ and $I_{825}(x, y)$ and $I_{980}(x, y)$ are the normalized sample image pixel value for the 825 and $980 \mathrm{~nm}$ wavelength images, respectively.

Finally for the artificial neural network (ANN) method, the Matlab Neural Network Toolbox was used for a three-layer Back-propagation Neural Network with the hidden and output layers having tangent-sigmoidal (tansig) and logarithmic-sigmoidal (logsig) processing functions, respectively. After pre-examining networks with 5, 10 and 20 neurons in the hidden layer the best network having the least mean square error was found with five neurons in the hidden layer. To train the network, the relative reflectance values from same selected points of the LDA formed the twoneuron input layer, with a bi-level single neuron output.

Binary bruise map image was generated using any of the above three methods. Knowing that bruises should appear sufficient in size, and to remove spurious and noisy bruised pixels, the bruise map image was morphologically opened with a five-pixel radius disc-structuring element. The effectiveness of the judgment methods was then assessed by visually tallying the results of judgment in a comparison matrix.

\section{Effects of storage}

After finishing the spectral imaging, the samples were placed in several plastic mesh boxes enclosed by another outer shell box that had shallow standing water inside. To achieve a high humidity environment, the boxes were covered with perforated plastic sheets and placed in a five-degree centigrade refrigerated storage. In the morning, all samples were withdrawn at least two $\mathrm{h}$ prior to a spectral imaging session to allow them to equilibrate with ambient conditions. Spectral images were taken at $0,1,2,3$, and 4 days after bruising application.

\section{RESULTS AND DISCUSSION}

\section{Sample and spectral profile}

From Table 1, the means of the mass of samples of the two maturity groups were found not significantly different (by Student's t-test), such that the effects of size differences could be factored out, and the two maturity groups could be adequately compared.

Looking at the spectral profile in Fig. 4, maturity difference was distinct at $675 \mathrm{~nm}$, the wavelength where the absorption peak of chlorophyll occurs (McGlone et al., 2002; Merzlyak et al., 2003). Additionally, bruised tissues had consistently lower relative reflectance from about $720 \mathrm{~nm}$ with a maximum difference occurring at the valley between 960 and $990 \mathrm{~nm}$. This range is noted for light absorption by water (McGlone et al., 2002; Shrestha et al., 2002; Zwiggelaar et al., 1996). NIR light can penetrate deeper into the fruit tissues than visible light and known to be easily absorbed by water. Bruising causes expulsion of cellular materials and water out into the extracellular spaces that increases the local light absorption in the NIR region and particularly at around 960-980 nm. Furthermore, because of the effects of pigmentation (chlorophyll and anthocyanin) at the visible 650 to $720 \mathrm{~nm}$ range, the detection of bruises within this range would seemed to be

Table 1 Profile of mass of samples at two levels of ripeness maturity.

\begin{tabular}{lccccc}
\hline Level of ripeness & $\mathrm{N}$ & Mean $^{\text {ns }}, \mathrm{gm}$ & $\mathrm{SD}, \mathrm{gm}$ & Maximum, gm & Minimum, gm \\
\hline Full-ripe & 60 & 17.43 & 2.25 & 21.70 & 12.54 \\
70-80\% ripe & 60 & 17.71 & 2.42 & 23.12 & 13.51 \\
\hline All samples & 120 & 17.57 & 2.33 & 23.12 & 12.54 \\
\hline
\end{tabular}

${ }^{\mathrm{ns}}$ not significantly different by Student's t-test. 


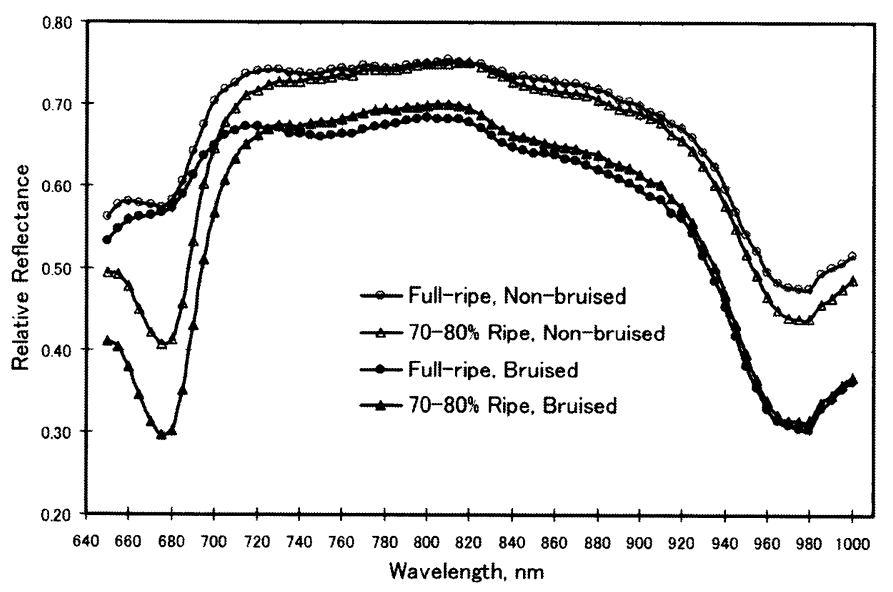

Fig. 4 Spectra of $\bigcirc$ full-ripe and non-bruised, $\triangle 70-80 \%$ ripe and non-bruised, $\bigcirc$ full-ripe and bruised, and $\boldsymbol{\Delta} 70-80 \%$ ripe and bruised and non-bruised strawberry fruit tissues. Relative diffuse reflectance values were computed from bruised and non-bruised parts of the fruits with respect to a white reference tile from images that were bandpass filtered from 650 to $1,000 \mathrm{~nm}$.

difficult and un-reliable. Figure 5 validates this while showing RGB camera-acquired image (in grayscale) and hyperspectral imaging-acquired image at $980 \mathrm{~nm}$. Figure 6 shows that the bruises distinctly appear well at $3.0 \mathrm{~N}$ force level, while the effect of ripeness maturity is easily seen at 1.5 $\mathrm{N}$ force level.

\section{Selection of wavelengths for bruise detection}

Table 2 shows the classification efficiencies of several discriminant function models (having different number of wavelengths) for both development and validation sample points. As the reflectance spectra suggests, the $980 \mathrm{~nm}$ comprise the single-wavelength model. While suitable as a least-cost option, difficulties can be introduced by temporal variations in the imaging system such as those caused by aging of components. A significant efficiency improvement was achieved with the second model ( $825 \mathrm{~nm}$ added), but little improvements thereafter. Since lesser costs at higher efficiencies are desired, the $980 \mathrm{~nm}$ and $825 \mathrm{~nm}$ wavelength pair seemed to give the best option.

From the analysis, the discriminant function using the data from the 825 and $980 \mathrm{~nm}$ wavelengths was:

$$
\mathrm{D}(x, y)=-22.818 \mathrm{R}_{825}(x, y)+34.930 \mathrm{R}_{980}(x, y)+3.019
$$

where $\mathrm{D}(x, y)$ is the discriminant score, such that if $\mathrm{D}(x, y)$ is less than zero, the pixel point at $(x, y)$ is considered bruised; $\mathrm{R}_{825}(x, y)$ and $\mathrm{R}_{980}(x, y)$ are the relative reflectance values at $825 \mathrm{~nm}$ and 980 $\mathrm{nm}$, respectively. Note that the reflectance at $825 \mathrm{~nm}$ was near to a peak $(800-820 \mathrm{~nm}$, Fig. 4), while the $980 \mathrm{~nm}$ marks the absorption band by water as explained earlier. These wavelengths were within reasonable correlation to the results $(860$ and $960 \mathrm{~nm})$ of the work of Shrestha et al. (2002). From Table 2, the non-bruised pixels were classified with very high accuracy (at least 99\%), and that ensured minimal false-positive classifications. For bruised pixels, the classification accuracy was also good due to the fact that in masking off the bruised areas, there was no absolute certainty that the points were actually bruised.

\section{Development of judgment model}

Figure 6 shows some resulting images of the judgment using LDA, while Table 3 shows the comparison matrix of the results. Consistent with LDA model development, the non-bruised $(0 \mathrm{~N})$ samples were perfectly classified as non-bruised. Similarly, bruised samples receiving $2.0 \mathrm{~N}$ and $3.0 \mathrm{~N}$ forces were perfectly classified due to high levels of damage. However, there were 


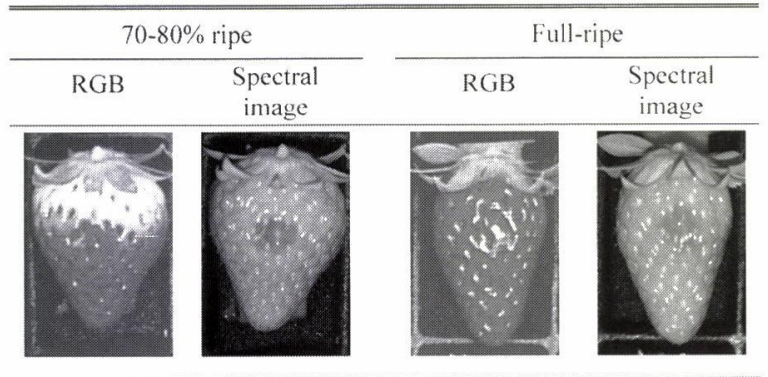

Fig. 5 RGB image (in grayscale) and spectral image at $980 \mathrm{~nm}$ for samples receiving $3.0 \mathrm{~N}$ bruise.

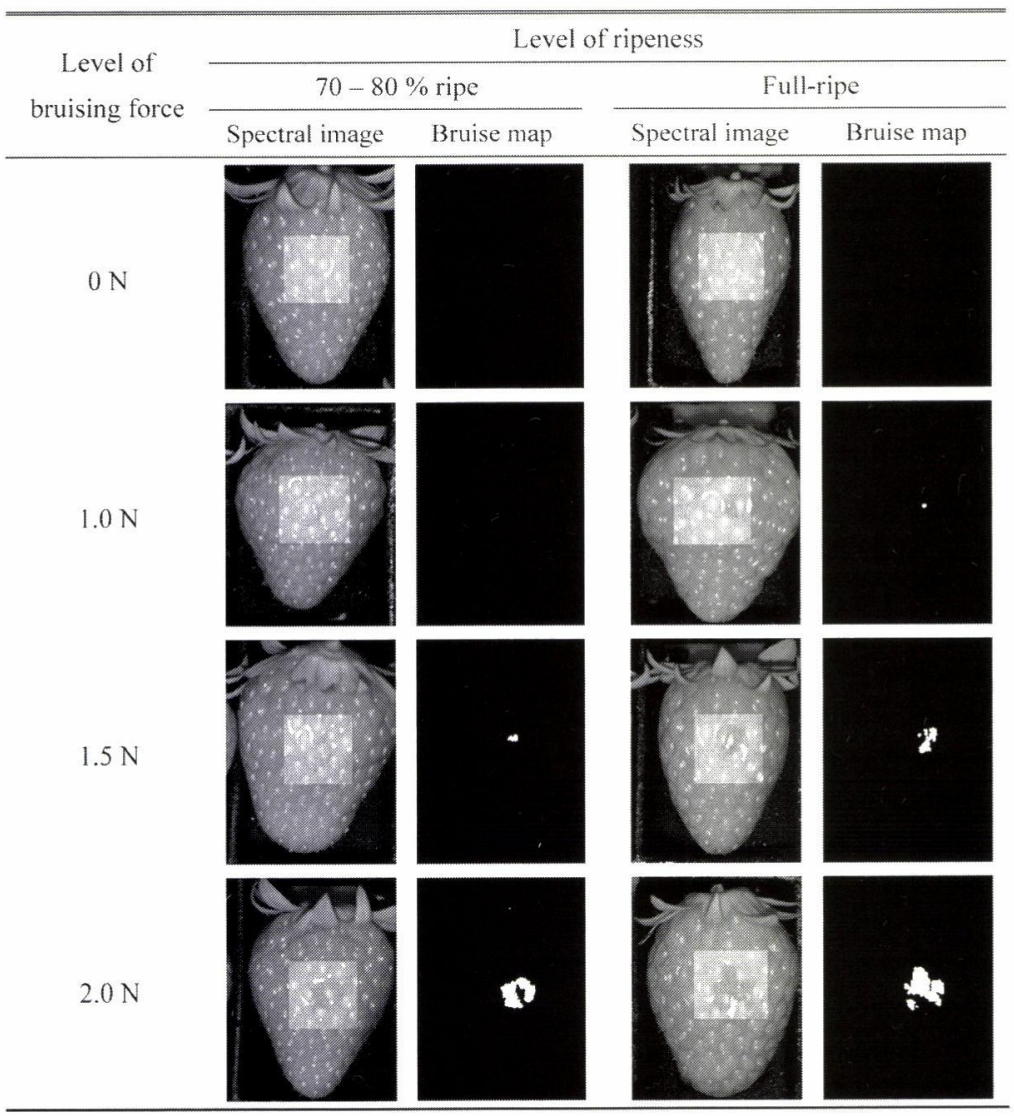

Fig. 6 Some spectral images at $980 \mathrm{~nm}$ and bruise maps detected using linear discriminant analysis judgment method on strawberry fruit samples.

differences in accuracies in other force levels of bruising force according to fruit ripeness maturity. At $0.5 \mathrm{~N}$ bruising force, few samples were classified as bruised. At $1.0 \mathrm{~N}$ bruising force, there was significantly more number of samples classified as bruised for the full-ripe level maturity than at $70-80 \%$ ripeness maturity. Finally at $1.5 \mathrm{~N}$ bruising force, the two levels of maturity had similar judgment results.

One important aspect of this study is identifying the threshold level of bruising force. At $70^{-}$ 
Table 2 Classification efficiency (success rates in \%) for bruised and non-bruised pixels using different models selected by stepwise linear discriminant analysis.

\begin{tabular}{lccccc}
\hline \multirow{2}{*}{$\begin{array}{l}\text { Discriminant function } \\
\text { wavelengths, } \mathrm{nm}\end{array}$} & \multicolumn{2}{c}{ Model development } & & \multicolumn{2}{c}{ Model validation } \\
\cline { 2 - 3 } \cline { 5 - 6 } & Non-bruised & Bruised & & Non-bruised & Bruised \\
\hline 980 & 96.0 & 88.4 & & 98.8 & 84.6 \\
980,825 & 99.4 & 90.7 & & 99.7 & 86.5 \\
$980,825,925$ & 99.4 & 90.9 & & 99.8 & 86.5 \\
$980,825,925,680$ & 99.6 & 91.3 & & 99.8 & 86.7 \\
$980,825,925,680,650$ & 99.5 & 91.8 & & 99.9 & 86.1 \\
\hline
\end{tabular}

Table 3 Comparison matrix for detection of bruises in strawberries by LDA, ND and ANN judgment methods.

\begin{tabular}{|c|c|c|c|c|c|c|c|c|c|c|c|c|}
\hline \multirow{3}{*}{$\begin{array}{l}\text { Bruising } \\
\text { force }\end{array}$} & \multicolumn{6}{|c|}{$70-80 \%$ ripe level } & \multicolumn{6}{|c|}{ Full-ripe level } \\
\hline & \multicolumn{3}{|c|}{ Bruised } & \multicolumn{3}{|c|}{ Non-bruised } & \multicolumn{3}{|c|}{ Bruised } & \multicolumn{3}{|c|}{ Non-bruised } \\
\hline & $\mathrm{LDA}^{2}$ & $\mathrm{ND}^{y}$ & $\mathrm{ANN}^{\mathrm{x}}$ & LDA & ND & ANN & LDA & ND & ANN & LDA & ND & ANN \\
\hline $0 \mathrm{~N}$ & $0^{\mathrm{u}}$ & 0 & 0 & 10 & 10 & 10 & 0 & 0 & 0 & 10 & 10 & 10 \\
\hline $0.5 \mathrm{~N}$ & 1 & 1 & 2 & 9 & 9 & 8 & 1 & 2 & 4 & 9 & 8 & 6 \\
\hline $1.0 \mathrm{~N}$ & 2 & 3 & 4 & 8 & 7 & 6 & 9 & 10 & 10 & 1 & 0 & 0 \\
\hline $1.5 \mathrm{~N}$ & 9 & 9 & 10 & 1 & 1 & 0 & 9 & 9 & 10 & 1 & 1 & 0 \\
\hline $2.0 \mathrm{~N}$ & 10 & 10 & 10 & 0 & 0 & 0 & 10 & 10 & 10 & 0 & 0 & 0 \\
\hline $3.0 \mathrm{~N}$ & 10 & 10 & 10 & 0 & 0 & 0 & 10 & 10 & 10 & 0 & 0 & 0 \\
\hline
\end{tabular}

${ }^{2}$ Linear discriminant analysis.

${ }^{y}$ Normalized difference.

"Artificial neural network.

" Numbers indicate the number of judged samples out of 10 samples in a set.

$80 \%$ ripe maturity level, the number of bruised samples had changed between $1.0 \mathrm{~N}$ and $1.5 \mathrm{~N}$, which indicates the threshold bruising force for this ripeness maturity. Similarly, for the full-ripe maturity level, the transition and hence the threshold bruising force, had occurred between $0.5 \mathrm{~N}$ and $1.0 \mathrm{~N}$. Expectedly, this observation had quantitatively shown that different levels of ripeness would have different levels of bruising susceptibility.

The ND method removes variations caused by complicating factors during image acquisition such as varying light levels, imaging geometry and aging components. One advantage of this technique is that the pair of wavelengths can be either logically selected based on an observation or ad hoc analysis, or from previous research. Utilizing the same wavelengths $(825$ and $980 \mathrm{~nm})$ found in LDA, bruised areas in ND images appeared brighter. Various threshold values were explored ( 0.28 to 0.32 ), and an optimum threshold for bruising was found at 0.30 based on the highest successful classification rate and visual examination. Generally, similar patterns of results were obtained from this method (Table 3). However, more samples were actually classified as bruised at of $0.5 \mathrm{~N}$ and $1.0 \mathrm{~N}$ bruising force levels, which might indicate an improvement over LDA.

The ANN method is generally better because of its capability to linearly and non-linearly map input variables to output results in classifying pixels. Using the same wavelengths of LDA and ND methods, the results of the performance of this technique are also shown in Table 3 . With marked similarity to the results of LDA, bruised samples were perfectly detected even up to $1.5 \mathrm{~N}$ level. An even more number of samples were identified as bruised for the $0.5 \mathrm{~N}$ and $1.0 \mathrm{~N}$ levels.

Figure 7 compares the performance of the three methods showing that the ANN method had detected a wider extent of bruising followed by the ND method. All three methods had performed well with better performance observed for the more severe bruising cases. The ANN method appeared better than the ND and LDA methods. However, this method is strongly dependent on the training dataset and the condition under which they were taken. Similarly for the statistically robust LDA method, different discriminant functions are needed for significant changes in, for 


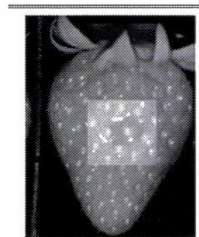

Original

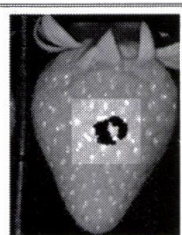

$\mathrm{LDA}^{\prime}$

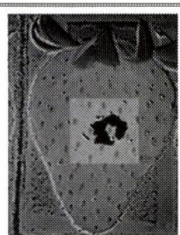

$\mathrm{ND}^{y}$

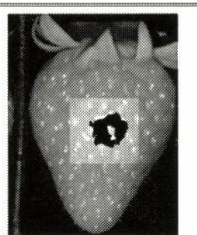

$\mathrm{ANN}^{\times}$

Fig. 7 Bruises on a strawberry fruit sample detected using three methods of judgment on a $70-80 \%$ ripe level strawberry receiving a $2.0 \mathrm{~N}$ bruising force.

linear discriminant analysis.

normalized difference.

artificial neural network.

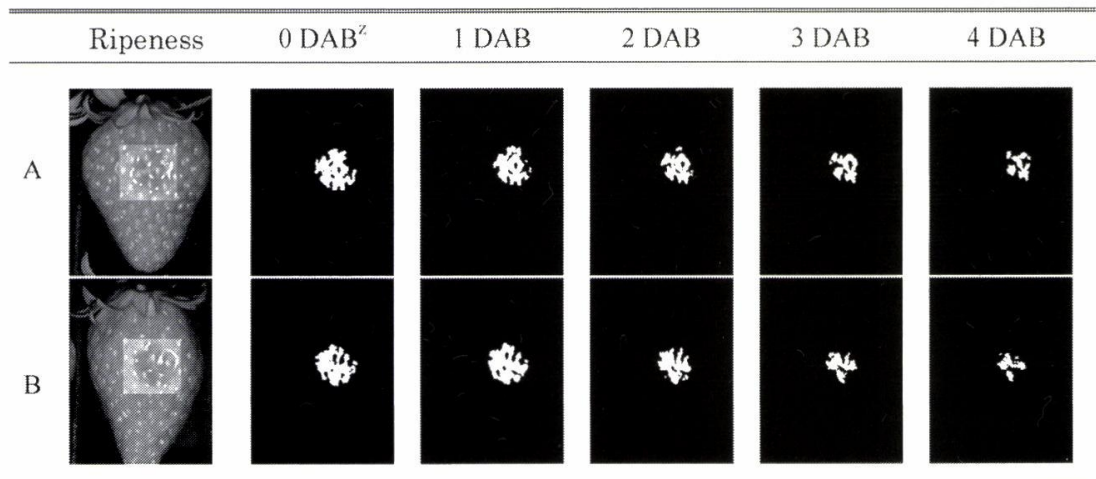

"days after bruising

Fig. 8 Reduction of extent of detected bruising with time for a (A) $70-80 \%$ ripe and (B) full-ripe level strawberry.

example, lighting conditions and geometry of acquisition. Finally, the ND method seemed extremely useful with its performance midway between the other two. Its computation is so simple that makes it quite more attractive to use especially for high-speed on-line operations.

4. Effects of storage time

Figure 8 shows examples of detected bruises over storage, whose duration simulated the time for fruit inspection. There was an observable reduction of the extent of detected bruised pixel points over time. Earlier researches (Lu, 2003; Abbott, 1999; Upchurch et al., 1994) had mostly attributed this to the loss of moisture from the fruits. The observation suggests that, firstly, the slightly bruised fruits (i.e. with low bruising force) seemed to recover from the damage, and secondly, the extent of detected bruising is affected by age of bruising. Unfortunately because the time and level of bruising are usually unknown, the exact severity of damage at the time of occurrence can never be fully known. What is more important is that bruises can be adequately detected at the time of inspection. However since slightly bruised fruits can seem to recover from the damage with time, these fruits may be judged acceptable.

In conclusion, the LDA of hyperspectral imaging spectral data of points had identified $825 \mathrm{~nm}$ and $980 \mathrm{~nm}$ wavebands for classifying pixel points between bruised and non-bruised on strawberries. Using this technique on the strawberry samples coupled with some image processing operations had yielded good judgment results. Using the same wavebands for ND and ANN, similar trends of classification accuracies were obtained. Despite that ANN had yielded the highest 


\section{NAGATA ET AL.}

accuracy for the detection of bruises, the ND method was found more useful because of its robustness and simplicity. Both new and old bruises can be detected by the spectral imaging, and there was a reduction of the extent of detected bruises observed with time.

This research was carried out in part through the Grant-In-Aid for Scientific Research (B)(2) No. 15380175, Ministry of Education, Culture, Sports, Science, and Technology. We also wish to acknowledge Mr. Mochihara of Kibana for providing the strawberry samples and Dr. Chi N. Thai of University of Georgia, Athens for comments.

\section{REFERENCES}

Abbott, J. A. 1999. Quality measurements of fruits and vegetables. Postharvest Biol. Tech. 15: 207-225.

Ariana, D. P., Shrestha, B. P., Guyer, D. E. 2003. Integrating reflectance and fluorescence imaging for apple disorder classification. ASAE Paper No. 033120. ASAE, St. Joseph, MI.

Cogdill, R. P., Hurburgh, C. R. Jr., Rippke, G. R. 2004. Single-kernel maize analysis by near-infrared hyperspectral imaging. Trans. ASAE 47: 311-320.

Evans, M. D., Thai, C. N., Grant, J. C. 1998. Development of a spectral imaging system based on a liquid crystal tunable filter. Trans. ASAE 41: $1845-1852$.

Hancock, J. F. 1999. Strawberries. CABI Publishing, New York, pp 237.

Kim, M. S., Chen, Y. R., Mehl, P. M. 2001. Hyperspectral reflectance and fluorescence imaging system for food quality and safety. Trans. ASAE 44: 721-729.

Lu, R. 2003. Detection of bruises on apples using near-infrared hyperspectral imaging. Trans. ASAE 46: 523-530.

McGlone, V. A., Jordan, R. B., Martinsen, P. J. 2002. Vis/NIR estimation at harvest of pre- and post-storage quality indices for 'Royal Gala' apple. Postharvest Biol. Tech. 25: 135-144.

Merzlyak, M. N., Solovchenko, A. E., Gitelson, A. A. 2003. Reflectance spectral features and nondestructive estimation of chlorophyll, carotenoid and anthocyanin content in apple fruit. Postharvest Biol. Tech. 27: 197-211.

Shrestha, B. P., Nagata, M., Gejima, Y. 2002. Study on image processing for quality estimation of strawberries: (Part 2) Detection of bruises on fruit by NIR image processing. J. SHITA 14: 1-9.

Upchurch, B. L., Throop, J. A., Aneshansley, D. J. 1994. Influence of time, bruise-type, and severity on nearinfrared reflectance from apple surfaces for automatic bruise detection. Trans. ASAE 37: 1571-1575.

Xing, J., Landahl, S., Lammertyn, J., Vrindts, E., De Baerdemaeker, J. 2003. Effects of bruise type on discrimination of bruised and non-bruised 'Golden Delicious' apples by VIS/NIR spectroscopy. Postharvest Biology Tech. 30: 249-258.

Zwiggelaar, R., Yang, Q. S., Garcis-Pardo, E., Bull, C. R. 1996. Use of spectral information and machine vision for bruise detection on peaches and apricots. J. Agric. Eng. Res. 63: 323-332. 